\title{
Article \\ The Two-Way Interaction between the Molecules That Cause Vaginal Malodour and Lactobacilli: An Opportunity for Probiotics
}

\author{
Scarlett Puebla-Barragan 1,2,*(D), Polycronis Paul Akouris 1,2 ${ }^{\mathbb{D}}$, Kait F. Al 1,2 (D), Charles Carr 1,2 (D), Britney Lamb ${ }^{1,2}$, \\ Mark Sumarah ${ }^{3} \mathbb{D}$, Charlotte van der Veer ${ }^{4}$, Remco Kort ${ }^{5,6}{ }^{\mathbb{D}}$, Jeremy Burton ${ }^{1,2} \mathbb{D}$ and Gregor Reid ${ }^{1,2} \mathbb{D}$
}

check for updates

Citation: Puebla-Barragan, S.; Akouris, P.P.; Al, K.F.; Carr, C.; Lamb, B.; Sumarah, M.; van der Veer, C.; Kort, R.; Burton, J.; Reid, G. The Two-Way Interaction between the Molecules That Cause Vaginal Malodour and Lactobacilli: An Opportunity for Probiotics. Int. J. Mol Sci. 2021, 22, 12279. https://doi.org/ 10.3390/ijms222212279

Academic Editors: Jerome

F. Strauss III and Kimberly

K. Jefferson

Received: 28 September 2021

Accepted: 6 November 2021

Published: 13 November 2021

Publisher's Note: MDPI stays neutral with regard to jurisdictional claims in published maps and institutional affiliations.

Copyright: (c) 2021 by the authors. Licensee MDPI, Basel, Switzerland. This article is an open access article distributed under the terms and conditions of the Creative Commons Attribution (CC BY) license (https:/ / creativecommons.org/licenses/by/ $4.0 /)$.
1 Canadian Centre for Human Microbiome and Probiotics, Lawson Health Research Institute, 268 Grosvenor Street, London, ON N6A 4V2, Canada; pakouris@uwo.ca (P.P.A.); kal@uwo.ca (K.F.A.); charlie.carr@mail.utoronto.ca (C.C.); blamb5@uwo.ca (B.L.); Jeremy.Burton@lawsonresearch.com (J.B.); gregor@uwo.ca (G.R.)

2 Departments of Microbiology and Immunology, Surgery, and Biochemistry, Western University, London, ON N6A 4V2, Canada

3 Agriculture and Agri-Food Canada, London, ON N5V 4T3, Canada; mark.sumarah@canada.ca

4 Department of Infectious Diseases, Public Health Service (GGD), Nieuwe Achtergracht 100, 1018 WT Amsterdam, The Netherlands; cvanderveer@mlw.mw

5 Department of Molecular Cell Biology, Faculty of Science, O2 Lab Building, Vrije Universiteit Amsterdam, De Boelelaan 1108, 1081 HZ Amsterdam, The Netherlands; r.kort@vu.nl

6 ARTIS-Micropia, Plantage Kerklaan 38-40, 1018 CZ Amsterdam, The Netherlands

* Correspondence: spueblab@uwo.ca

Abstract: Vaginal malodour is a sign of dysbiosis. The biogenic amines (BAs) cadaverine, putrescine and tyramine are known to be causative compounds. Recent reports suggest these compounds produced by pathogens might have a role beyond causing malodour; namely inhibiting the growth of lactobacilli bacteria that are crucial in the maintenance of vaginal homeostasis. The aim of this study was to identify whether certain lactobacilli strains could reduce BAs and to evaluate how Lactobacillus species were affected by these compounds. Using LC-MS and HPLC-UV, five Lactobacillus crispatus strains were identified as being capable of significantly reducing BAs from the media under in vitro conditions. Through $16 \mathrm{~S}$ rRNA gene sequencing of vaginal swabs exposed to Bas, cadaverine was found to reduce the relative abundance of lactobacilli. When L. crispatus was exposed to media supplemented with BAs with an $\mathrm{HCl}$ adjusted lower $\mathrm{pH}$, its growth was enhanced, demonstrating the relevance of the maintenance of an acidic vaginal environment. If strains are to be developed for probiotic application to alleviate bacterial vaginosis and other conditions affecting large numbers of women worldwide, their ability to adapt to Bas and regulate $\mathrm{pH}$ should be part of the experimentation.

Keywords: vaginal malodour; dysbiosis; probiotics; lactobacilli; vaginal microbiota; biogenic amines

\section{Introduction}

The biogenic amines (BAs) cadaverine, putrescine, and tyramine, have been correlated with malodour in the urogenital tract of women with dysbiosis. Patients with conditions such as bacterial vaginosis (BV) and urinary tract infection (UTI) suffer from malodour, negatively impacting their quality of life [1,2]. Unfortunately, the gold standard treatment for both conditions is a course of antibiotics. However, these do not target malodour, raising the concept that conjoint treatment could be developed.

Probiotics, defined as 'live microorganisms that, when administered in adequate amounts, confer a health benefit on the host' $[3,4]$, have been used for decades to help manage and prevent dysbiosis of the female urogenital tract. Studies primarily using Lacticaseibacillus rhamnosus GR-1 (LGR-1) and Limosilactobacillus reuteri RC-14 (LRC-14) have shown benefits in regulating homeostasis [5-8]. Indeed, LGR-1 is the most widely 
studied probiotic for urogenital health $[5,6]$, and its genome indicates that it can thrive in the vaginal environment $[5,6]$. When used in conjunction with LRC-14 it can decrease recurrence of BV, UTI and effectively inhibit the yeast Candida albicans, which is one of the most frequent vaginal pathogens [7-9]. Additionally, the combination taken orally during and following antibiotic treatment aids in the replenishment of indigenous species such as Lactobacillus crispatus, which are predominant in most healthy vaginas [10]. This suggests that lactobacilli-based probiotics could be used, in combination with antibiotics, to address malodour. Nonetheless, not all probiotics are the same, and their health benefits must be well documented. As such, it was our goal to identify which strains could potentially aid in the reduction of malodorous compounds and to provide a better understanding of the mechanisms involved.

Previous studies have suggested that BAs are produced by some pathogens and they act as virulence factors that increase the urogenital $\mathrm{pH}$, reduce production of lactic acid, and lower the abundance of lactobacilli $[11,12]$. Previously, several strains of L. crispatus have exhibited the potential to degrade BAs [13]. In the present study, five clinical isolates of L. crispatus were tested for their ability to reduce BAs, along with the type-strain L. crispatus ATCC 33820, LGR-1, and LRC-14. The relationship between these malodorous compounds and lactobacilli survival was also examined. We hypothesized that the interaction between lactobacilli and BAs is key for the maintenance of vaginal homeostasis. The long-term goal is to determine if probiotics represent a novel approach to manage urogenital dysbiosis and malodour.

\section{Results}

\subsection{Lactobacilli Growth in Biogenic Amines}

When grown in VDMP supplemented with $100 \mu \mathrm{g} / \mathrm{mL}$ of cadaverine, putrescine, or tyramine as independent experiments, only L. crispatus ATCC 33820 showed a statistically significant reduction of cadaverine and putrescine. Strain LGR-1 produced a significant amount of tyramine when grown by itself, but this was tempered when LRC-14 was added. LRC14 showed a decreasing trend in the amounts of cadaverine and putrescine, but statistical significance was not reached. Results are summarized in Figure 1.

A Cadaverine

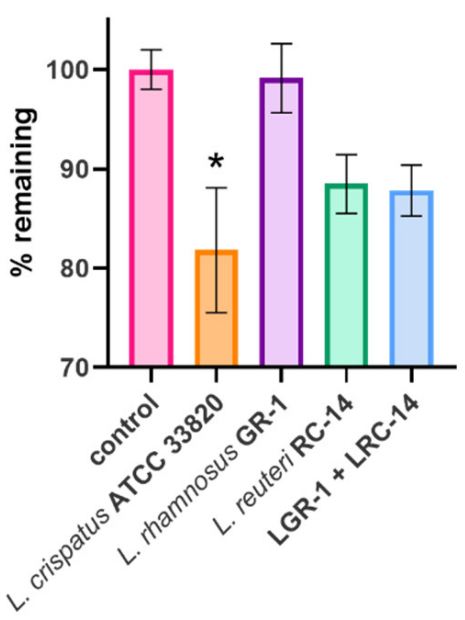

B

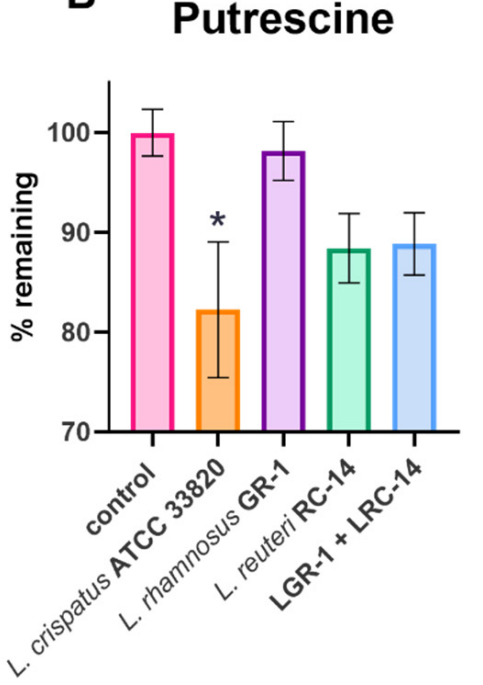

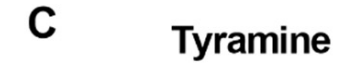

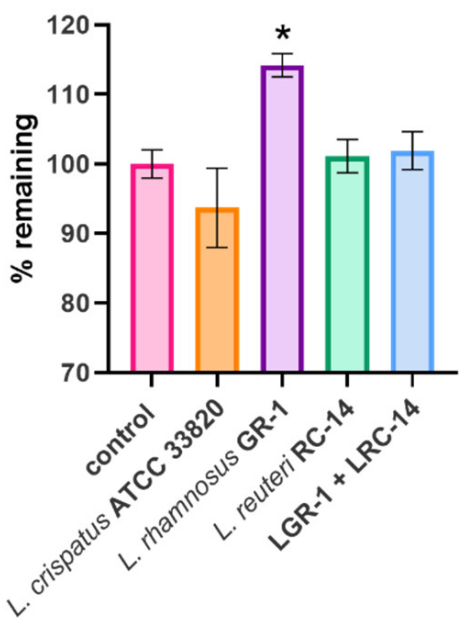

Figure 1. Metabolism of biogenic amines by different lactobacilli strains. Control consisted of media supplemented with each BA of interest. Bar plots show the remaining concentration of (A) cadaverine, (B) putrescine, and (C) tyramine, after $24 \mathrm{~h}$ of incubation with each strain of interest. Data are presented as means of 4 independent experiments $\pm 95 \%$ confidence intervals (CI). One-way ANOVA with the Dunnet correction for multiple comparisons was used to calculate statistical significance, $\left.{ }^{*} p \leq 0.05\right)$. 
Figure 2 shows that $L$. crispatus clinical isolates could significantly reduce the concentration of putrescine and cadaverine when supplemented with $200 \mu \mathrm{g} / \mathrm{mL}$ each, with the exception of strain RL12, which increased the amount of cadaverine significantly. Furthermore, all strains completely removed tyramine from the media. No biogenic amines were detected in any of the negative controls.

A

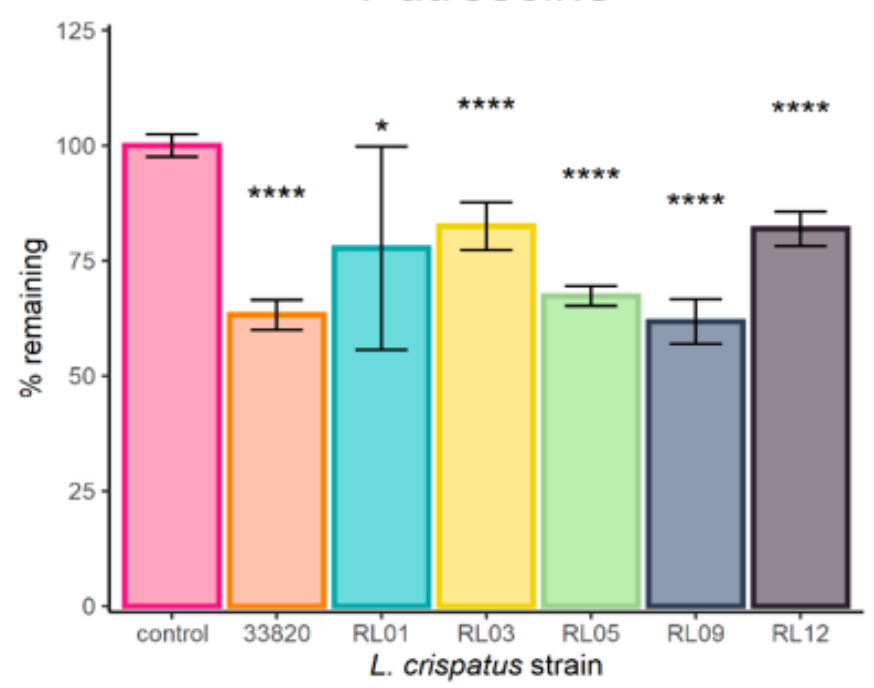

B

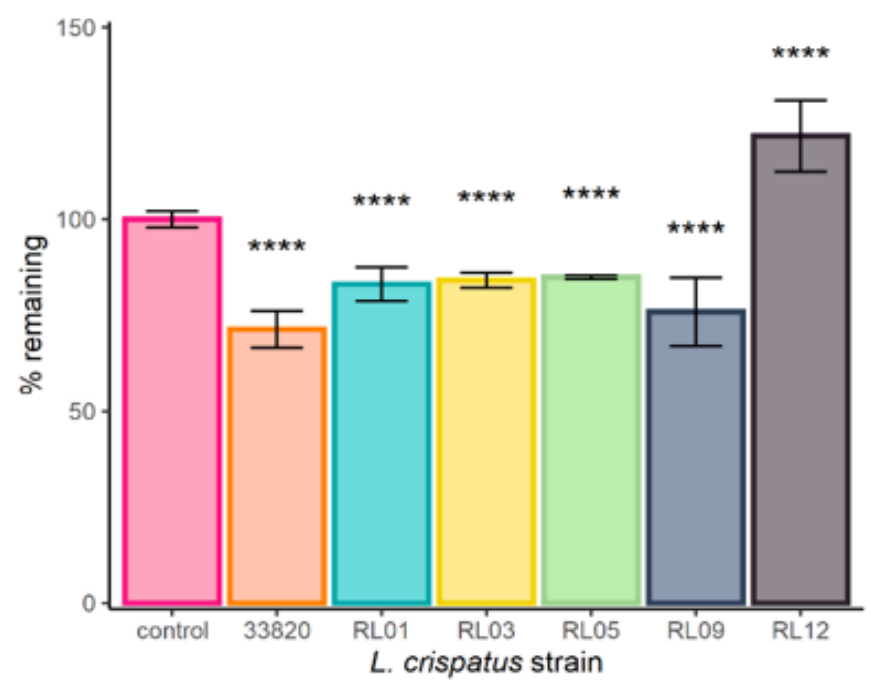

Figure 2. Metabolism of biogenic amines by different L. crispatus strains in amine VDMP. Control consists of amine VDMP only (no bacteria). Bar plots show the remaining concentration of (A) cadaverine and (B) putrescine, after $24 \mathrm{~h}$ of incubation with each strain of interest, One-way ANOVA with the Dunnet correction for multiple comparisons was used to calculate statistical significance ${ }^{*} p \leq 0.05$ and $\left.{ }^{* * *} p \leq 0.0001\right)$. Data are presented as means of 3 independent experiments with two technical replicates each $\pm 95 \%$ CI.

When amine-reduction assays were carried out using cultures of L. crispatus ATCC 33820 that had been originally exposed to putrescine, cadaverine, and tyramine (i.e., 'induced' cultures), all preparations from either whole cells or supernatants reduced the amount of all tested amines below $60 \%$. There was no statistically significant difference between induction and treatments for reduction ability of cadaverine. In the case of putrescine, 'induced' bacterial cultures showed further amine depletion than other treatments. Both the whole cells and the supernatant of 'induced' cultures reduced significantly more tyramine. Results are presented in Figure 3.

Due to the physicochemical properties of TMA, none of the analytical methods (i.e., LC-MS/MS and HPLC-UV) were suitable for detecting this compound at relevant concentrations. Therefore, it was excluded. 

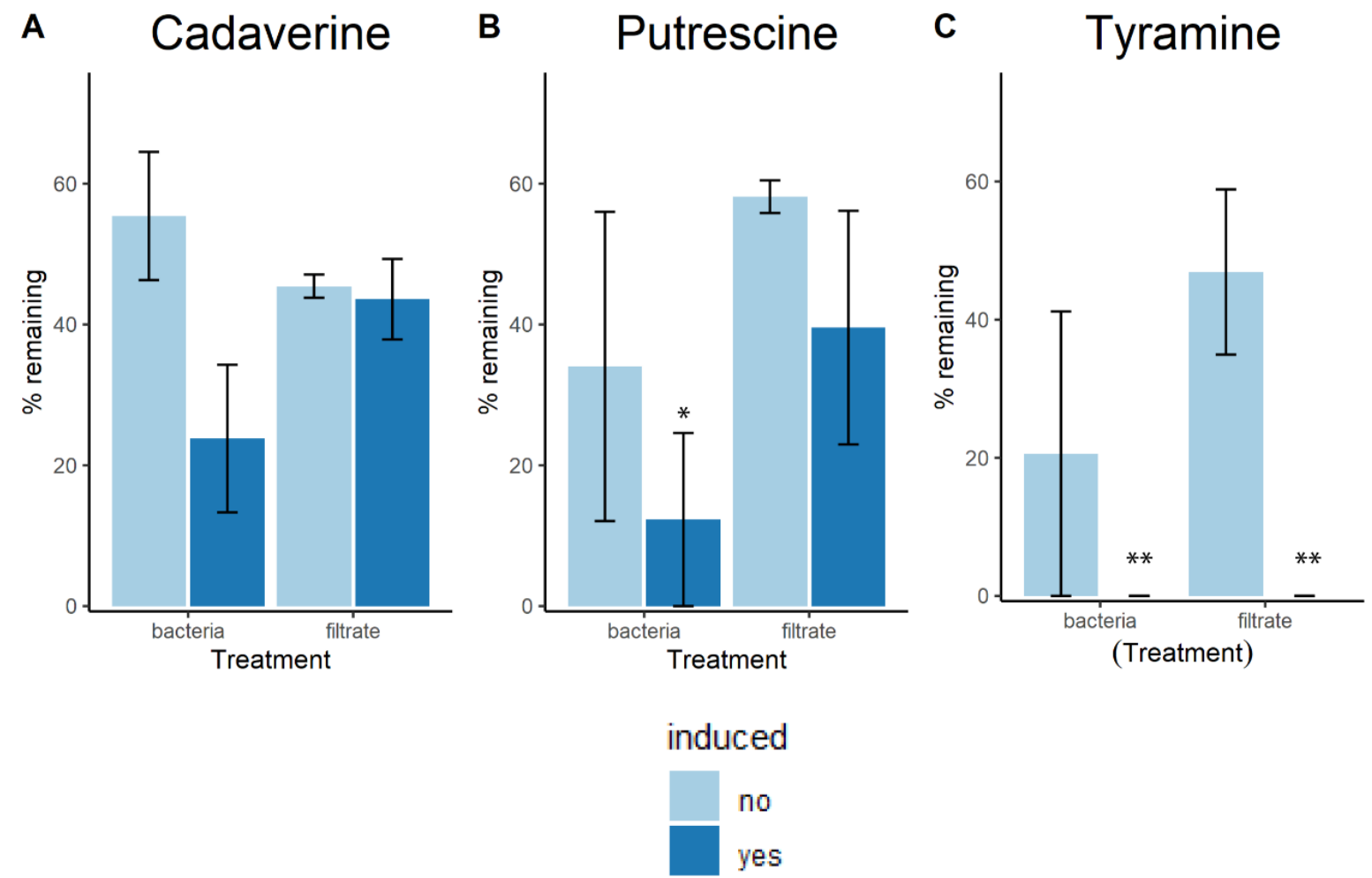

Figure 3. Biogenic amines exposure assay. Bar plots show the remaining concentration of (A) cadaverine, (B) putrescine, and $(C)$ tyramine, after incubation with each amine of interest. One-way ANOVA with the Tukey post hoc test for multiple comparisons was used to calculate statistical significance $\left({ }^{*} p \leq 0.05\right.$ and $\left.{ }^{* *} p \leq 0.01\right)$. Data are presented as means of 3 independent experiments two technical replicates each $\pm 95 \% \mathrm{CI}$.

\subsection{Effect of Biogenic Amines and $p H$ on the Growth of Lactobacillus Crispatus}

Originally, the $\mathrm{pH}$ of non-amine-supplemented VDMP (control) was 6.71 and that of BA media was 7.04. An additional set of each media was prepared with adjusted $\mathrm{pH}$ that corresponded to its counterpart (i.e., control at 7.04 and BA-media at 6.71). At the end of the incubation period all treatments reached a final $\mathrm{pH}$ of 4.50 .

Results showed that both 'induced' and 'uninduced' cultures had the most and fastest growth when placed in BA-VDMP that had been adjusted to a lower $\mathrm{pH}$ (Figure 4). They were followed by 'induced' and 'uninduced' cultures grown in VDMP at its original $\mathrm{pH}$ (6.71). Next, the bacteria were grown in BA-media at its original pH (7.04); 'induced' cultures showed better growth than their 'uninduced' counterparts. The slowest and lowest growths were observed for strains in VDMP at an adjusted higher $\mathrm{pH}$ (7.04). Statistical analyses of different relevant growth parameters between groups are summarized in Supplementary Tables S1-S8.

Analyses of the impact of exposure to biogenic amines on vaginal microbiota are shown in Figure 5. Results revealed that Lactobacillus abundance was significantly reduced when cultures were exposed to $200 \mu \mathrm{g} / \mathrm{mL}$ of cadaverine or to media supplemented with biogenic amines at similar concentrations to those found in vaginal secretions during BV [11,12]. 

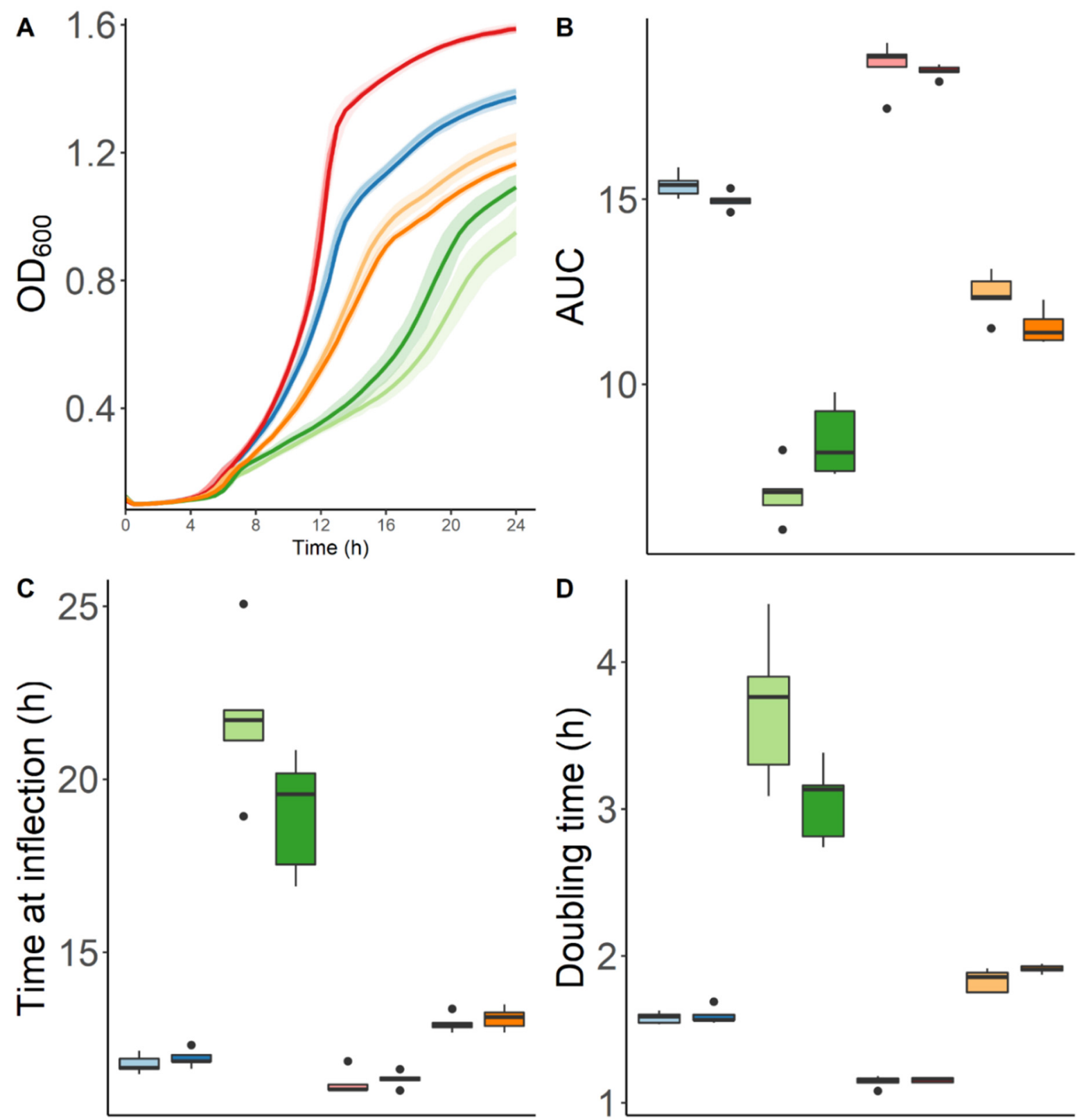

Treatment

- Induced control, $\mathrm{pH}=6.71$

- Uninduced control, $\mathrm{pH} 6.71$

- Induced control, $\mathrm{pH}=7.04$

- Uninduced control, $\mathrm{pH}=7.04$

- Induced BA media, $\mathrm{pH}=6.71$

- Uninduced BA media, $\mathrm{pH}=6.71$

Induced BA media, $\mathrm{pH}=7.04$

- Uninduced BA media, $\mathrm{pH}=7.04$

Figure 4. Effect of biogenic amines and $\mathrm{pH}$ on the growth of L. crispatus ATCC 33820. Data are presented as the means of 5 independent experiments. (A) Bacterial growth curves measured by absorbance at a wavelength of $600 \mathrm{~nm}$. (B) Logistic areas under the curve. (C) Time at inflection. (D) Doubling times. 

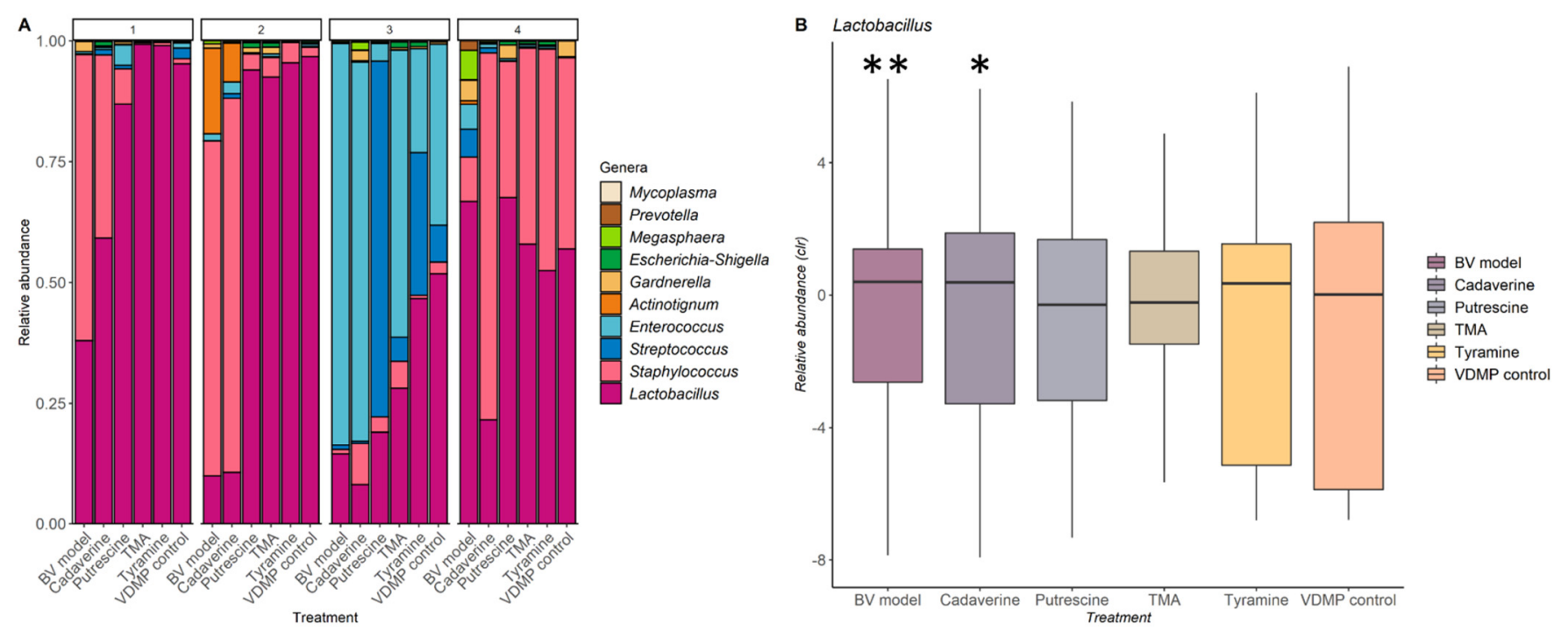

Figure 5. Impact of biogenic amines on the vaginal microbiota. (A) Bar plot of relative abundances. Each colour represents a genus and top numbers indicate each subject from which the sample originated. $X$-axis denotes which treatment each swab underwent. (B) Relative abundances of Lactobacillus spp. in different media (centered log ratios (CLR) are plotted). $\left({ }^{*} p \leq 0.05\right.$ and $\left.{ }^{* *} p \leq 0.01\right)$.

\section{Discussion}

Probiotic strains LRC-14 and LGR-1 showed some reduction of cadaverine and putrescine concentrations (both individually and as a coculture). Interestingly, LGR-1 significantly increased tyramine; however, when cocultured with LRC-14 this effect was not observed. These two strains are administered in combination, and these findings suggests a further benefit of this cooperation [14-18].

We previously characterized several clinical L. crispatus strains in terms of their potential to degrade and/or produce biogenic amines, while all of them have the genes for both functions, only a few were able to reduce the amount of amines in growth media. This formed the basis for selecting the strains for the present study [13]. Of significant note, L. crispatus ATCC 33820 and five vaginal L. crispatus isolates showed statistically significant reduction of putrescine, cadaverine and tyramine within $24 \mathrm{~h}$. The exception was strain RL12 which increased tyramine. Cultures of the type strain which had been either exposed ('induced') or not ('uninduced') to biogenic amines prior to the experiment resulted in reduced concentration of BAs from the media. Furthermore, bacteria originating from 'induced' cultures had a higher capacity to reduce the concentration of amines, better resistance to high $\mathrm{pH}$, and they grew better in the presence of amines. Similarly, filtered supernatants from 'uninduced' and especially 'induced' cultures were able to reduce the concentration of amines. It is unclear if this adaptation is $\mathrm{pH}$ - or metabolite-mediated. Previous studies have shown that the expression of the arginine deiminase pathway (which plays a major role in the metabolism of cadaverine and putrescine [13]) is pH-dependent in other Lactobacillus species [19]. Future studies should focus on global metabolomics and transcriptomic analyses that might identify which metabolic pathways are different between both groups and could therefore be causing these differences.

Interestingly, as shown in Figure 4A, when the high pH caused by BA was adjusted, L. crispatus ATCC 33820 grew better than in the medium alone. This novel finding suggests that under low $\mathrm{pH}$ conditions, lactobacilli can use biogenic amines as nutrient sources. This raises the question of whether in some women who experience malodour that self-resolves, the indigenous $L$. crispatus have been able to adapt and reassert themselves.

It has been previously shown that when growth medium is acidified via the addition of $\mathrm{HCl}$, as was the case in our study, the specific growth rate of lactobacilli and the production of lactic acid are slowed down [20]. However, the presence of metabolizable sugars has a 
protective role and enhances survival in L. rhamnosus GG [21]. Our results suggest that this could also be true of biogenic amines, which become bioavailable energy sources at lower $\mathrm{pH}$. Further studies are required using different minimal growth media supplemented with BAs to predict the metabolic pathways in which the biogenic amines are being used by the bacteria. In addition, this could verify if this effect is translatable to various lactobacilli strains, or, if it is a phenomenon specific for L. crispatus spp. Specifically, transcriptomic and metabolomic studies are warranted to better understand the metabolic adaptations that might occur as responses to changes in the $\mathrm{pH}$ of the environment.

The buffering capacity of vaginal fluids has been previously suggested as an indicator of reproductive health [22]. Thus, approaches that aid in the maintenance of an acidic environment, such as the use of prebiotics [23], are highly relevant to regulate the concentration of biogenic amines, thereby preventing dysbiosis and malodour.

Previous observations determined that biogenic amine exposure slowed down and reduced the growth capacity of lactobacilli [12]. However, we identified that this inhibitory effect can be reversed depending on the $\mathrm{pH}$ conditions. This might explain why some lactobacilli strains can produce small amounts of biogenic amines to support the growth of others, thereby maintaining an acidic environment that prevents the proliferation of pathogenic bacteria [13]. A limitation of our study is that we cannot infer causality from our observations. In the future, multiomics analyses should further characterize the phenomena we observed and aim to better understand the mechanisms behind them.

The microbiota composition study of vaginal samples showed that at concentrations of biogenic amines like those found in the vaginal fluids of patients with $\mathrm{BV}$, the relative abundance of Lactobacillus spp. is reduced and associated with an increase in $\mathrm{pH}$. This, in turn, allows for further proliferation of pathogens towards a state of dysbiosis. Furthermore, only media containing cadaverine had the ability to significantly decrease the proportion of Lactobacillus spp., suggesting that this compound plays a key role in the infection process and onset of malodour. The impact of other amines is not clear and should be further explored with larger sample sizes.

Overall, these findings provide a strong rationale for assessing candidate probiotic lactobacilli strains for their capacity to degrade biogenic amines and regulate $\mathrm{pH}$. This study highlights the relevance of maintaining a low $\mathrm{pH}$ in the vaginal environment, since this can turn biogenic amines from inhibitory compounds into energy sources that support the growth of lactobacilli.

\section{Materials and Methods}

\subsection{Lactobacillus Crispatus Clinical Strains}

The L. crispatus strains used in this study were selected based on their previously determined ability to reduce the concentration of BAs [13] and their genetic potential to degrade BAs. Strains were provided by van der Veer et al. [24] with all material approved by each participant with written consent and approval from the ethics review board of the Academic Medical Center (AMC), University of Amsterdam, The Netherlands. They originated from vaginal swabs obtained from women attending for a health check at the Sexually Transmitted Infections Clinic in Amsterdam, The Netherlands. The samples came from two groups of women: one with healthy vaginal microbiota (based upon Nugent score 0-3), or those with dysbiosis (Nugent score 7-10). The swabs were plated on modified trypticase soy agar and incubated anaerobically at $37^{\circ} \mathrm{C}$ for $24 \mathrm{~h}$. Next, single colonies underwent $16 \mathrm{~S}$ rRNA gene sequencing for identification purposes. The strains were then cryopreserved and stored at $-80^{\circ} \mathrm{C}$ in vaginally defined medium plus peptone (VDMP) [25]. Additional strains were obtained in London, Ontario, Canada from four healthy premenopausal women aged between 25 and 30 years old who consented to partake in the study, approved by the Health Sciences Research Ethics Board at The University of Western Ontario (File number 106089, original approval date 13 January 2015) [23]. The subjects self-swabbed their lateral vaginal walls using sterile Dacron swabs. Vaginal $\mathrm{pH}$ was confirmed to be approximately 4.5 by using $\mathrm{pHem}$-Alert applicator keys 
(Gynex Corporation, Redmond, WA, USA). Swabs were Gram-stained (Becton, Dickinson \& Company, Franklin Lakes, NJ, USA) and scored 0-3 using the Nugent system [26,27].

\subsection{Biogenic Amine Reduction Experiments}

An amine-reduction assay using LGR-1, LRC-14, and L. crispatus ATCC 33820 was performed, along with a polymicrobial culture containing LGR1 and LRC-14. Four individual colonies of each organism were grown in de Man-Rogosa-Sharpe (MRS) broth medium (VWR) at $37^{\circ} \mathrm{C}$ anaerobically for $24 \mathrm{~h}$. Next, $10 \mu \mathrm{L}$ of the initial liquid cultures was grown for $24 \mathrm{~h}$ anaerobically at $37^{\circ} \mathrm{C}$ in $10 \mathrm{~mL}$ of VDMP supplemented with $100 \mu \mathrm{g} / \mathrm{mL}$ of either cadaverine, putrescine, and tyramine (Sigma Aldrich, St. Louis, MO, USA) or a control of each specific amine media without bacteria to control for aerial effect of the volatile compounds. These were subsequently analyzed using liquid chromatography-mass spectrometry (LC-MS/MS).

To test their ability to degrade amines at similar concentrations to those in vaginal dysbiosis [28], three individual colonies of five L. crispatus strains (i.e., RL01, RL03, RL05, RL09, and RL12) as well as the type strain (ATCC 33820) were grown in MRS medium at $37^{\circ} \mathrm{C}$ anaerobically for $24 \mathrm{~h}$. Then, $10 \mu \mathrm{L}$ was subcultured in $2 \mathrm{~mL}$ of either VDMP or amine-VDMP $(200 \mu \mathrm{g} / \mathrm{mL}$ of cadaverine, $200 \mu \mathrm{g} / \mathrm{mL}$ of putrescine, and $200 \mu \mathrm{g} / \mathrm{mL}$ of tyramine) (Sigma Aldrich, St. Louis, MO, USA) and grown anaerobically at $37^{\circ} \mathrm{C}$ for $24 \mathrm{~h}$. The control consisted of amine-VDMP or VDMP with no bacteria. These samples were analysed using high performance liquid chromatography coupled to an ultraviolet detector (HPLC-UV).

\subsection{Induction Assays}

To identify whether L. crispatus undergoes adaptation after exposure to BAs, five individual colonies of L. crispatus ATCC 33820 were grown in $2 \mathrm{~mL}$ of amine-VDMP anaerobically at $37^{\circ} \mathrm{C}$ for $24 \mathrm{~h}$, then $10 \mu \mathrm{L}$ was inoculated into $2 \mathrm{~mL}$ of amine-VDMP under the same conditions; these were deemed the 'induced' group. Meanwhile, the 'uninduced' group was derived from 5 individual colonies of L. crispatus ATCC 33820 grown in $2 \mathrm{~mL}$ of VDMP anaerobically at $37^{\circ} \mathrm{C}$ for $24 \mathrm{~h}$, and subsequently subcultured in amine-VDMP. Next, HPLC-UV analysis was performed.

To assess whether the metabolites produced by L. crispatus had the ability to degrade BAs, the spent media of the original liquid cultures was filter sterilized $(0.22 \mu \mathrm{m})$ and incubated under the same conditions as the previous samples in amine-VDMP. These groups were designed 'induced' and 'uninduced' filtrates.

The effect of $\mathrm{pH}$ on the tolerance to amines of 'induced' and 'uninduced' cultures was measured by inoculating a 1:100 dilution of the liquid cultures in $240 \mu \mathrm{L}$ of BA-media (amine-VDMP $+50 \mu \mathrm{g} / \mathrm{mL}$ of TMA). The control consisted of VDMP only $(n=5)$. The $\mathrm{pH}$ was adjusted using either $\mathrm{NaOH}$ or $\mathrm{HCl}$. Absorbance at $600 \mathrm{~nm}$ was measured every $30 \mathrm{~min}$ over $24 \mathrm{~h}$ in an automatic plate reader. Finally, $\mathrm{pH}$ was measured using $\mathrm{pH}$ strips (Eorta). The R package growthcurveR [29] was used to model the bacterial growth kinetics and to calculate relevant growth parameters (i.e., maximum growth capacity, area under the curve, time at inflection point, and doubling time).

Data analysis was performed based on linear models generated for each growth parameter. Families were defined as either induced or uninduced. Within each family, multiple comparisons and control for type I error were performed using Tukey's post hoc test (Supplementary Tables S1, S3, S5 and S7). Next, individual contrasts between both families at every pH were performed (Supplementary Tables S2, S4, S6 and S8).

\subsection{LC-MS/MS Protocol}

After incubation, $250 \mu \mathrm{L}$ of each liquid culture was aliquoted and diluted 1:3 with pure methanol then vortexed and incubated on ice for $30 \mathrm{~min}$, followed by centrifugation at $16,000 \times g$ for $10 \mathrm{~min}$. Supernatants were filtered into vials for analysis $(0.22 \mu \mathrm{m}$ PTFE syringe filters) which was carried out using a Q-Exactive Quadrupole Orbitrap MS, cou- 
pled to an Agilent 1290 HPLC system with an Agilent HILIC-Z $(2.1 \times 100 \mathrm{~mm}, 2.7 \mu \mathrm{m})$. The mobile phase A consisted of $20 \mathrm{mM}$ ammonium formate in water, and phase $\mathrm{B}$ was $20 \mathrm{mM}$ ammonium formate in $90 \%$ acetonitrile. Analytes were eluted with the following gradient: $0 \mathrm{~min}, 100 \% \mathrm{~B} ; 0.5 \mathrm{~min}, 100 \% \mathrm{~B} ; 5.3 \mathrm{~min}, 80 \% \mathrm{~B} ; 9.5 \mathrm{~min}, 30 \% \mathrm{~B} ; 13.5 \mathrm{~min}, 30 \%$ B; $14.5 \mathrm{~min} 100 \% \mathrm{~B}$; and $16.5 \mathrm{~min}, 100 \% \mathrm{~B}$. Conditions for heated electrospray ionization (HESI) were as follows: HESI (+) capillary voltage, capillary voltage, $3.5 \mathrm{kV}$; capillary temperature, $250{ }^{\circ} \mathrm{C}$; sheath gas, 30.00 units; auxiliary gas, 8.00 units; probe heater temperature, $450{ }^{\circ} \mathrm{C}$; S-Lens RF level, 60.00. The instrument was operated with a top-3 data-dependent acquisition protocol with a full MS scan in the mass range of $\mathrm{m} / \mathrm{z} 1.2$ and collision energy of 28. Authentic standards were used for calibration curves (cadaverine, putrescine, and tyramine), acquired from Sigma-Aldrich (St. Louis, MO, USA). Compound identification and quantification was performed using the software Xcalibur (Thermo Fisher Scientific, Waltham, MA, USA).

\subsection{HPLC-UV Analysis}

Bacterial cultures and their supernatants were prepared by diluting them 1:10 with water, incubating them on ice for $30 \mathrm{~min}$ and centrifuging at $16,000 \times g$ for $10 \mathrm{~min}$. Samples were prepared for derivatization by adding $250 \mu \mathrm{L}$ of $0.5 \mathrm{KH}_{2} \mathrm{PO}_{4}$ buffer $(\mathrm{pH}=11)$ and $10 \mu \mathrm{L}$ of $1 \mathrm{M} \mathrm{NaOH}$. Next, $500 \mu \mathrm{L}$ of p-toluenesulfonyl chloride $(10 \mathrm{mg} / \mathrm{mL}$, Sigma Aldrich, St. Louis, MO, USA) was added and samples were incubated at $56{ }^{\circ} \mathrm{C}$ for $10 \mathrm{~min}$. The reaction was stopped with the addition of $50 \mu \mathrm{L}$ of $1 \mathrm{M} \mathrm{HCl}$ and samples were filtered into vials (0.45 $\mu$ L PTFE syringe filters) [30].

Analysis was carried out using a mobile phase in isocratic mode, composed of $60 \%$ acetonitrile and 40\% HPLC-grade water (Thermo Fisher Scientific, Waltham, MA, USA). The flow rate was $1 \mathrm{mg} / \mathrm{mL}$. Authentic standards for putrescine, cadaverine, and putrescine were used, and $\alpha$-aminobutyric acid was used as an internal standard, all acquired from Sigma-Aldrich. Analyte identification and quantification was performed using the Chemstation software B.04.03 (Agilent, Santa Clara, CA, USA).

\subsection{Analysis of the Impact of Biogenic Amines on the Vaginal Microbiota}

To assess the impact that biogenic amines have on the vaginal microbiota, an adaptation of the in vitro, polymicrobial, vaginal culturing model described previously was used [23]. Briefly, bacteria collected from vaginal swabs were grown anaerobically for $36 \mathrm{~h}$ in different media: VDMP supplemented with either $200 \mu \mathrm{g} / \mathrm{mL}$ of cadaverine, $200 \mu \mathrm{g} / \mathrm{mL}$ of putrescine, $200 \mu \mathrm{g} / \mathrm{mL}$ of tyramine, or $50 \mu \mathrm{g} / \mathrm{mL}$ of TMA, BA-VDMP, and VDMP. Different media were prepared to properly evaluate the effect of each amine alone and as a combination. The concentrations of amines were selected based on what is normally found in vaginal fluids of patients with BV.

Microbiota analysis was performed by amplification of the $\mathrm{V} 4$ region of $16 \mathrm{~S}$ ribosomal RNA, which was then sequenced using the Illumina MiSeq (San Diego, CA, USA) to detect shifts in microbial abundance [31]. Earth Microbiome universal primers, 515F and 806R, were used for PCR amplification. Primers consisted of an Illumina adapter and four random nucleotides, one of 24 unique 12-mer barcodes, and the corresponding annealing primer [32]. A Biomek ${ }^{\circledR} 3000$ Laboratory Automation Workstation (Beckman-Coulter, Mississauga, ON, Canada) was used for PCR set-up. Amplification was performed in an Eppendorf thermal cycler (Eppendorf, Mississauga, ON, Canada) with an initial rise in temperature of $95{ }^{\circ} \mathrm{C}$, then 25 cycles of one minute each at $95^{\circ} \mathrm{C}, 52{ }^{\circ} \mathrm{C}$, and $72{ }^{\circ} \mathrm{C}$. Purified amplicons were then paired-end sequenced with 250 cycles on an Illumina MiSeq platform (San Diego, CA, USA) [32].

Data were exported as raw fastq files. Quality control was performed following the DADA2 pipeline [32]. Taxonomy was assigned using the SILVA (c132) training set, and reads corresponding to Eukaryota, Mitochondria, and Chloroplast were removed [32]. Amplicon sequence variants (SVs) present at $\geq 1 \%$ relative abundance were maintained. 
Downstream analysis was performed with the ALDEx2 [33-35], Vegan [36], rstatix [37], and emmeans [38] R packages.

\subsection{Statistical Analyses}

All data analyses were performed using RStudio. One-way analysis of variances (ANOVAs) and all post hoc tests were conducted with the R packages rstatix v0.7.0 [37]. To correct heteroscedasticity, marginal means were used and the matrix of covariance was adjusted. This was performed using the R packages emmeans v1.6.0 [38] and sandwich v3.0-1 [39]. Figure 1 was created using the software GraphPad Prism v.8. The rest of the figures were made with the R package ggplot2 [40].

Supplementary Materials: The following are available online at https://www.mdpi.com/article/ 10.3390/ijms222212279/s1. Table S1: Effect of biogenic amine supplemented media on maximum possible population (K). Table S2: Effect of previous amine exposure on maximum possible population (K). Table S3: Effect of biogenic amine supplemented media on time at inflection. Table S4: Effect of previous amine exposure on time at inflection. Table S5: Effect of amine supplemented media on doubling time. Table S6: Effect of previous amine exposure on doubling time. Table S7: Effect of amine supplemented media on logistic area under the curve. Table S8: Effect of previous amine exposure on logistic area under the curve.

Author Contributions: Conceptualization, S.P.-B. and G.R.; methodology, S.P.-B., K.F.A., C.C., C.v.d.V., M.S. and R.K.; software, S.P.-B., K.F.A. and C.C.; formal analysis, S.P.-B., K.F.A. and C.C.; investigation, S.P.-B., K.F.A., C.C., P.P.A., C.v.d.V., B.L., G.R.; resources, G.R., R.K., M.S. and J.B.; writing—original draft preparation, S.P.-B.; writing—review and editing, G.R., K.F.A., B.L., P.P.A., C.v.d.V., M.S., R.K., J.B.; visualization, S.P.-B.; supervision, G.R.; project administration, G.R. and S.P.-B.; funding acquisition, G.R., S.P.-B., R.K., M.S. and J.B. All authors have read and agreed to the published version of the manuscript.

Funding: S.P.-B. is funded by the Mexican Council of Science and Technology, grant number: CVU 692895.

Institutional Review Board Statement: The research proposal for this study was assessed by the ethics review board of the Academic Medical Center (AMC), University of Amsterdam, The Netherlands. The review board deemed that no additional ethical approval was required for this study, since the vaginal samples used here were collected as part of routine procedure for cervical examinations at the STI clinic in Amsterdam (document reference number W12_086 \# 12.17.0104). The collection of vaginal swabs used for metagenomic analysis was approved the Health Sciences Research Ethics Board at The University of Western Ontario (File number 106089, original approval date 13 January 2015).

Informed Consent Statement: Informed consent was obtained from all subjects involved in the study. Clients of the STI clinic were notified that their samples could be used for scientific research, after anonymization of client clinical data and samples. The data and samples of clients who refused were discarded. This procedure was approved by the AMC ethics review board (reference number W15_159 \# 15.0193).

Data Availability Statement: The data for this study have been deposited in the European Nucleotide Archive (ENA) at EMBL-EBI under accession number PRJEB48740.

Acknowledgments: We want to thank Justin Renaud and Shannon Senney for their technical assistance. As well as the members of the Burton and Reid labs, and our collaborators in Amsterdam.

Conflicts of Interest: The authors state no conflict of interest, bar G.R. who consults for Seed.

\section{References}

1. McMillan, A.; Rulisa, S.; Sumarah, M.; Macklaim, J.M.; Renaud, J.; Bisanz, J.E.; Gloor, G.B.; Reid, G. A multi-platform metabolomics approach identifies highly specific biomarkers of bacterial diversity in the vagina of pregnant and non-pregnant women. Sci. Rep. 2015, 5, 14174. [CrossRef]

2. Subramanian, C.; Nyirjesy, P.; Sobel, J.D. Genital malodor in women: A modern reappraisal. J. Low. Genit. Tract Dis. 2012, 16, 49-55. [CrossRef] 
3. FAO/WHO Working Group. Report of a Joint FAO/WHO Expert Consultation on Evaluation of Health and Nutrition Properties of Probiotics in Food Including Powder Milk with Live Lactic acid Bacteria; FAO: Cordoba, Argentina, 2002; ISSN 0254-4725. Available online: http:/ / www.fao.org/3/a0512e/a0512e.pdf (accessed on 1 June 2021).

4. Hill, C.; Guarner, F.; Reid, G.; Gibson, G.R.; Merenstein, D.J.; Pot, B.; Morelli, L.; Canani, R.B.; Flint, H.J.; Salminen, S.; et al. The International Scientific Association for Probiotics and Prebiotics consensus statement on the scope and appropriate use of the term probiotic. Nat. Rev. Gastroenterol. Hepatol. 2014, 11, 506-514. [CrossRef] [PubMed]

5. Petrova, M.I.; Reid, G.; Ter Haar, J.A. Lacticaseibacillus rhamnosus GR-1, a.k.a. Lactobacillus rhamnosus GR-1: Past and future perspectives. Trends Microbiol. 2021, 8, 747-761. [CrossRef] [PubMed]

6. Petrova, M.I.; Macklaim, J.M.; Wuyts, S.; Verhoeven, T.; Vanderleyden, J.; Gloor, G.B.; Lebeer, S.; Reid, G. Comparative genomic and phenotypic analysis of the vaginal probiotic Lactobacillus rhamnosus GR-1. Front. Microbiol. 2018, 9, 1278. [CrossRef]

7. Martinez, R.; Franceschini, S.A.; Patta, M.C.; Quintana, S.M.; Candido, R.C.; Ferreira, J.C.; De Martinis, E.C.P.; Reid, G. Improved treatment of vulvovaginal candidiasis with fluconazole plus probiotic Lactobacillus rhamnosus GR-1 and Lactobacillus reuteri RC-14. Lett. Appl. Microbiol. 2009, 48, 269-274. [CrossRef]

8. Vujic, G.; Jajac Knez, A.; Despot Stefanovic, V.; Kuzmic Vrbanovic, V. Efficacy of orally applied probiotic capsules for bacterial vaginosis and other vaginal infections: A double-blind, randomized, placebo-controlled study. Eur. J. Obstet. Gynecol. Reprod. Biol. 2013, 168, 75-79. [CrossRef]

9. Reid, G. Probiotic use in an infectious disease setting. Expert Rev. Anti-Infect. Ther. 2017, 15, 449-455. [CrossRef]

10. Macklaim, J.M.; Clemente, J.C.; Knight, R.; Gloor, G.B.; Reid, G. Changes in vaginal microbiota following antimicrobial and probiotic therapy. Microb. Ecol. Health Dis. 2015, 26, 27799. [CrossRef]

11. Borgogna, J.-L.C.; Shardell, M.D.; Grace, S.G.; Santori, E.K.; Americus, B.; Li, Z.; Ulanov, A.; Forney, L.; Nelson, T.M.; Brotman, R.M.; et al. Biogenic amines increase the odds of bacterial vaginosis and affect the growth of and lactic acid production by vaginal Lactobacillus spp. Appl. Environ. Microbiol. 2021, 87, e03068-20. [CrossRef] [PubMed]

12. Nelson, T.M.; Borgogna, J.-L.C.; Brotman, R.M.; Ravel, J.; Walk, S.T.; Yeoman, C.J. Vaginal biogenic amines: Biomarkers of bacterial vaginosis or precursors to vaginal dysbiosis? Front. Physiol. 2015, 6, 1-15. [CrossRef]

13. Puebla-Barragan, S.; Watson, E.; van der Veer, C.; Chmiel, J.A.; Carr, C.; Burton, J.P.; Sumarah, M.; Kort, R.; Reid, G. Interstrain variability of human vaginal lactobacillus crispatus for metabolism of biogenic amines and antimicrobial activity against urogenital pathogens. Molecules 2021, 26, 4538. [CrossRef]

14. Anukam, K.C.; Osazuwa, E.; Osemene, G.I.; Ehigiagbe, F.; Bruce, A.W.; Reid, G. Clinical study comparing probiotic Lactobacillus GR-1 and RC-14 with metronidazole vaginal gel to treat symptomatic bacterial vaginosis. Microbes Infect. 2006, 8, $2772-2776$. [CrossRef] [PubMed]

15. Reid, G.; Charbonneau, D.; Erb, J.; Kochanowski, B.; Beuerman, D.; Poehner, R.; Bruce, A.W. Oral use of Lactobacillus rhamnosus GR-1 and L. fermentum RC-14 significantly alters vaginal flora: Randomized, placebo-controlled trial in 64 healthy women. FEMS Immunol. Med. Microbiol. 2003, 35, 131-134. [CrossRef]

16. Martinez, R.; Seney, S.; Summers, K.; Nomizo, A.; De Martinis, E.; Reid, G. Effect of Lactobacillus rhamnosus GR-1 and Lactobacillus reuteri RC-14 on the ability of Candida albicans to infect cells and induce inflammation. Microbiol. Immunol. 2009, 53, 487-495. [CrossRef] [PubMed]

17. Reid, G.; Bruce, A.W.; Fraser, N.; Heinemann, C.; Owen, J.; Henning, B. Oral probiotics can resolve urogenital infections. FEMS Immunol. Med. Microbiol. 2001, 30, 49-52. [CrossRef] [PubMed]

18. Karlsson, M.; Scherbak, N.; Khalaf, H.; Olsson, P.E.; Jass, J. Substances released from probiotic Lactobacillus rhamnosus GR-1 potentiate NF-KB activity in Escherichia coli-stimulated urinary bladder cells. FEMS Immunol. Med. Microbiol. 2012, 66, 147-156. [CrossRef]

19. Rimaux, T.; Rivière, A.; Illeghems, K.; Weckx, S.; De Vuyst, L.; Leroy, F. Expression of the arginine deiminase pathway genes in Lactobacillus sakei is strain dependent and is affected by the environmental pH. Appl. Environ. Microbiol. 2012, 78, 4874-4883. [CrossRef]

20. Narendranath, N.V.; Power, R. Relationship between $\mathrm{pH}$ and medium dissolved solids in terms of growth and metabolism of lactobacilli and Saccharomyces cerevisiae during ethanol production. Appl. Environ. Microbiol. 2005, 71, 2239-2243. [CrossRef]

21. Corcoran, B.M.; Stanton, C.; Fitzgerald, G.F.; Ross, R.P. Survival of probiotic lactobacilli in acidic environments is enhanced in the presence of metabolizable sugars. Appl. Environ. Microbiol. 2005, 71, 3060-3067. [CrossRef]

22. Noguchi, T.; Sado, T.; Naruse, K.; Kobayashi, H. Vaginal fluid pH and buffer capacity for predicting false preterm labor in Japanese women. Int. J. Gynecol. Obstet. 2016, 134, 69-74. [CrossRef] [PubMed]

23. Collins, S.L.; McMillan, A.; Seney, S.; van der Veer, C.; Kort, R.; Sumarah, M.W.; Reid, G. Promising prebiotic candidate established by evaluation of lactitol, lactulose, raffinose, and oligofructose for maintenance of a Lactobacillus-dominated vaginal microbiota. Appl. Environ. Microbiol. 2017, 84, e02200-17. [CrossRef]

24. van der Veer, C.; Hertzberger, R.Y.; Bruisten, S.M.; Tytgat, H.L.P.; Swanenburg, J.; de Kat Angelino-Bart, A.; Schuren, F.; Molenaar, D.; Reid, G.; de Vries, H.; et al. Comparative genomics of human Lactobacillus crispatus isolates reveals genes for glycosylation and glycogen degradation: Implications for in vivo dominance of the vaginal microbiota. Microbiome 2019, 7, 49. [CrossRef] [PubMed]

25. Geshnizgani, A.M.; Onderdonk, A.B. Defined medium simulating genital tract secretions for growth of vaginal microflora. J. Clin. Microbiol. 1992, 30, 1323-1326. [CrossRef] [PubMed] 
26. Nugent, R.; Krohn, M.; Hillier, S. Reliability of diagnosing bacterial vaginosis is improved by a standardized method of Gram stain interpretation. J. Clin. Microbiol. 1991, 29, 297-301. [CrossRef] [PubMed]

27. Spiegel, C.; Amsel, R.; Holmes, K. Diagnosis of bacterial vaginosis by direct Gram stain of vaginal fluid. J. Clin. Microbiol. 1983, 18, 170-177. [CrossRef] [PubMed]

28. Wolrath, H.; Forsum, U.; Larsson, P.G.; Borén, H. Analysis of bacterial vaginosis-related amines in vaginal fluid by gas chromatography and mass spectrometry. J. Clin. Microbiol. 2001, 39, 4026. [CrossRef]

29. Sprouffske, K. Simple Metrics to Summarize Growth Curves. R Package Version 0.3.1. 2020. Available online: https:/ / cran.rproject.org/package=growthcurver/index.html (accessed on 25 July 2021).

30. Dziarkowska, K.; Jönsson, J.Á.; Wieczorek, P.P. Single hollow fiber SLM extraction of polyamines followed by tosyl chloride derivatization and HPLC determination. Anal. Chim. Acta 2008, 606, 184-193.b. [CrossRef]

31. Al, K.F.; Denstedt, J.D.; Daisley, B.A.; Bjazevic, J.; Welk, B.K.; Pautler, S.E.; Gloor, G.B.; Reid, G.; Razvi, H.; Burton, J.P. Ureteral stent microbiota is associated with patient comorbidities but not antibiotic exposure. Cell Rep. Med. 2020, 1, 100094. [CrossRef]

32. Parada, A.E.; Needham, D.M.; Fuhrman, J.A. Every base matters: Assessing small subunit rRNA primers for marine microbiomes with mock communities, time series and global field samples. Environ. Microbiol. 2016, 18, 1403-1414. [CrossRef]

33. Gloor, G.B.; Macklaim, J.M.; Fernandes, A.D. Displaying variation in large datasets: Plotting a visual summary of effect sizes. J. Comput. Graph. Stat. 2016, 25, 971-979. [CrossRef]

34. Fernandes, A.D.; Reid, J.N.; Macklaim, J.M.; McMurrough, T.A.; Edgell, D.R.; Gloor, G.B. Unifying the analysis of high-throughput sequencing datasets: Characterizing RNA-seq, 16S rRNA gene sequencing and selective growth experiments by compositional data analysis. Microbiome 2014, 2, 15. [CrossRef] [PubMed]

35. Fernandes, A.D.; Macklaim, J.M.; Linn, T.G.; Reid, G.; Gloor, G.B. ANOVA-like differential expression (ALDEx) analysis for mixed population RNA-seq. PLoS ONE 2013, 8, e67019. [CrossRef] [PubMed]

36. Oksanen, J.; Blanchet, G.; Friendly, M.; Kindt, R.; Legendre, P.; McGlinn, D.; Minchin, P.; O’Hara, R.; Simpson, G.; Solymos, P.; et al. Vegan: Community Ecology Package. 2020. Available online: http://cran.r-project.org/web/packages/vegan/index.html (accessed on 25 July 2021).

37. Kassambara, A. Rstatix: Pipe-Friendly Framework for Basic Statistical Tests. R Package Version 0.7.0. 2021. Available online: http:/ / cran.r-project.org/web/packages/rstatix/index.html (accessed on 25 July 2021).

38. Lenth, R. Emmeans: Estimated Marginal Means, Aka Least-Squares Means. R Package Version 1.6.0. 2021. Available online: http:/ / cran.r-project.org/web/packages/emmeans/index.html (accessed on 25 July 2021).

39. Zeileis, A. Econometric computing with HC and HAC covariance matrix estimators. J. Stat. Softw. 2004, 11, 1-17. [CrossRef]

40. Wickham, H. Ggplot2: Elegant Graphics for Data Analysis; Springer: New York, NY, USA, 2016; ISBN 978-0-387-98140-6. 